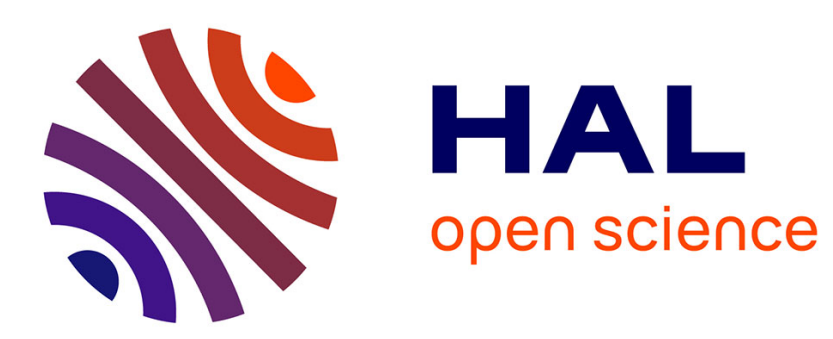

\title{
On the perturbation analysis of interactive buckling in nearly symmetric structures
}

\author{
Angelo Luongo, Marcello Pignataro
}

\section{To cite this version:}

Angelo Luongo, Marcello Pignataro. On the perturbation analysis of interactive buckling in nearly symmetric structures. International Journal of Solids and Structures, 1992, 29 (6), pp.721-733. hal00804680

\section{HAL Id: hal-00804680 \\ https://hal.science/hal-00804680}

Submitted on 26 Mar 2013

HAL is a multi-disciplinary open access archive for the deposit and dissemination of scientific research documents, whether they are published or not. The documents may come from teaching and research institutions in France or abroad, or from public or private research centers.
L'archive ouverte pluridisciplinaire HAL, est destinée au dépôt et à la diffusion de documents scientifiques de niveau recherche, publiés ou non, émanant des établissements d'enseignement et de recherche français ou étrangers, des laboratoires publics ou privés. 


\title{
ON THE PERTURBATION ANALYSIS OF INTERACTIVE BUCKLING IN NEARLY SYMMETRIC STRUCTURES
}

\author{
A. LuONGo \\ Dipartimento di Ingegneria delle Strutture. Universita di L'Aquilia. Monteluco Roio. \\ 67040 L'Aquila. Italia \\ and \\ M. Pignataro \\ Dipartimento di Ingegneria Strutturale e Geotecnica. Universita" di Roma "La Sapienza". \\ 00184 Roma, Italia
}

(Received 100 Octoher 1900) in revisced form 2 Sume 1991)

\begin{abstract}
Different perturbation methods for the analysis of non-lineatr interaction between simultancous buckling modes of nearly symmetric structures are discussed. First, the merturbation method employed by Budiansky for a single buckling mode, is exlended a consider modes interaction of a perfect structure, by determining both the slope and the curvature of the bifurcated paths. It is shown that the solution diverges. when a properly detined parameter which characteriaes the asymmetry of the structure approilches zero, thus preventing to recoser results of symmetric systems. A moditied perturbation mothod which permits to surmount this drawhick is then suggested : this method applies only to a cliss of structures and furnishes asymptotic series valid in a wide region around bifurcation. The two methods are applied to investigate the post-buckling hehavior af a two-degrec-of-frecelom system. linally, a novel perturbation methed which follows to some extent the lines of the (jalerkin method and is particularly powerful in the investigation of nearly symumetric systems is presented.
\end{abstract}

\section{INTRODUCTION}

Non-linear problems, both in statics and dynamics, are often treated with perturbation techniques which have the advantage of being easy to apply and able to furnish parametric solutions of the problems (Koiter, 1945; Budiansky, 1974; Nayfeh, 1973). The algorithm. when applied, for instance, to an equation of the type

$$
L\left(u ; \lambda_{0}\right)+c_{2} u^{2}+c_{3} u^{3}+\cdots=0
$$

where $L$ is a linear operator and $c_{2}, c_{3}$ are constants, consists in expressing the state variable $u$ and the control parameter $\lambda$ as a power series in terms of a perturbation parameter $\xi$

$$
\begin{aligned}
& u=\xi u_{1}+1 \xi^{2} u_{2}+\cdots \\
& \lambda=\lambda_{\mathrm{c}}+\xi \lambda_{1}+1 \xi_{2} \lambda_{2}+\cdots
\end{aligned}
$$

and in determining the coefficients of the series expansion through the solution of a sequence of linear perturbation equations.

Usually, the series expansion is truncated at the first term which is different from zero. For instance, in a bifurcation problem where $i$ denotes the load, the expansion is truncated at the linear terms if $\lambda_{1} \neq 0$, or at the quadratic terms if $\lambda_{1}=0$ and $\lambda_{2} \neq 0$. The equilibrium 
problems which are encountered in practice, manifest a number of buckling modes by correspondence with the same value of the critical load $\lambda_{c}$ (interactive buckling).

Interactive problems have been extensively investigated in the past years. The interest has in particular been focused on the analysis of thin-walled members under compression which may undergo local or overall buckling [see for example the papers by Byskov and Hutchinson (1979). Sridharan and Benito (1984). Bradford and Hancock (1984), Benito and Sridharan (1985), Sridharan and Ali (1985), Pignataro et al. (1985), Pignataro and Luongo (1987), Luongo and Pignataro (1989) and Byskov et al. (1989)] but little attention has been devoted to nearly symmetric structures.

In this paper we wish to analyze the post-buckling behavior of nearly-symmetric elastic systems which exhibit several buckling modes and show that some perturbation techniques lead to inaccurate results.

\section{PERTURBATION ANALYSIS OF SIMULTANEOUS BUCKLING MODES}

The equilibrium paths of an elastic structure subjected to conservative loads which exhibits a number of simultaneous buckling modes are determined. First. the standard perturbation analysis is applied by following the Budiansky (1974) formulation where the series expansion is carried out one step further; then a modified perturbation method is presented which is applicable to a particular class of systems. A brief sketch of the two procedures is presented here: details can be found in Pignataro and Luongo (1988) and Luongo and Pignataro (1988).

\subsection{Standard porturhation method}

Let us consider a hyperelastic body system subjected to conservative loads characterized by the total potential energy functional $w[w ; \lambda]$. where $w$ is the displacement field and $i$ a parameter governing the external force field acting on it. The equilibrium condition is obtained by requiring the functional $d,[11, \lambda]$ to be stationary with respect to kinematically admissible displacement fields, that is

$$
\omega^{\prime}\left[u^{\prime} ; \lambda\right] \dot{\delta} u^{\prime}=0 \quad \forall \dot{\delta} u^{\prime}
$$

where a prime denotes Frechet differentiation with respect to it:

In buckling problems it is assumed that at at certain critical value $\lambda_{c}$ of the load factor $i$, the state $w_{i}$ belongs to two diflerent equilibrium paths : the fundamental one $w_{0}(\lambda)$, which is taken to be known. and the bifurcated path $w(\lambda)$. In general, the fundamental path is no easier to tind than any other path. and in an analytical sense it is known a priori only for simple cases. However. in many problems, an approximate description of the fundamental path is sufficient for an adequate estimate of the post-critical behavior.

By introducing the differential state variable $u(\lambda)$, the bifurcated path can be described as

$$
u(\lambda)=u_{10}(\lambda)+u(\lambda)
$$

It is usually convenient to express the function $u(\lambda)$ using the parametric relations $u=u(\zeta), i=\lambda(\zeta)$ which are assumed to be regular. These relations can then be expressed through the series expansion (2), where $\xi=0$ corresponds to bifurcation. By substituting eqn (4) into (3). by expanding this equation in terms of $u$ and $\dot{\lambda}$ starting from $u=0$ and $i=\lambda_{c}$, respectively, and by using relations (2) we get

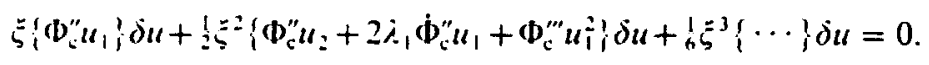


By equating separately to zero all terms with the same power of 3 . the following perturbation equations are obtained:

$$
\begin{aligned}
& \Phi_{\mathrm{L}}^{\prime \prime} u \mid j u=0 \\
& \Phi_{c}^{\prime \prime} u_{z} \dot{\partial} u=-\left\{2 \dot{j}_{1} \dot{\Phi}_{c}^{\prime \prime} u_{1}+\Phi_{i}^{\prime \prime} u_{i}^{i}\right\} \dot{\delta} u \\
& \Phi_{c}^{\prime \prime} u_{3} \delta u=-3\left\{\lambda_{1} \dot{\Phi}_{c}^{\prime \prime} u_{2}+\lambda_{2} \dot{\Phi}_{c}^{\prime \prime} u_{1}+i_{i} \ddot{\Phi}_{c}^{\prime \prime} u_{1}+\Phi_{c}^{\prime \prime \prime} u_{1} u_{2}+\lambda_{1} \dot{\Phi}_{i}^{\prime \prime \prime} u_{i}^{\prime}+\Phi_{c}^{\prime \prime \prime \prime} u_{1}^{\prime} j_{j} u\right.
\end{aligned}
$$

where

$$
\begin{aligned}
\Phi_{c}^{\prime \prime} & =\Phi^{\prime \prime}\left[w_{0}\left(\lambda_{c}\right) ; \lambda_{c}\right] \\
\dot{\Phi}_{c}^{\prime \prime} & =\left[\frac{\mathrm{d}}{\mathrm{d} \lambda} \Phi^{\prime \prime}\left[w_{0}(\lambda) ; \lambda\right]\right]_{i=i_{c}} \\
& =\left[\Phi^{\prime \prime \prime}\left[w_{0}(\lambda) ; \lambda\right] \frac{\mathrm{d}}{\mathrm{d} \lambda} w_{0}(\lambda)+\frac{i}{\partial \lambda}()^{\prime \prime}\left[w_{01}(\lambda) ; \lambda\right]\right]_{;} ;
\end{aligned}
$$

Analogous positions hold for higher order differentiations. Note that the procedure breaks down when bifurcation occurs at a limit load $\left(\mathrm{d} w_{0} / \mathrm{d} z=x\right)$ and therefore this calse will be excluded in the sequel.

Equation (fal) is an eigenvalue problem which is assumed to admit the multiple eigenvalue $\lambda_{s}$ and the $m$ eigenfunctions $r_{3}$. The first order displacements field may therefore be expressed as a lincar combination with arbitrary coellicients $\mu$, of the $m$ independent solutions

$$
u_{1}=\mu_{1} c_{1} \quad(i=1,2, \ldots m)
$$

After replacing eqn ( 8 ) into $(6 \mathrm{~b})$, coellicients $\mu$, are determined by imposing on the second

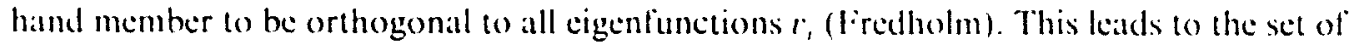
$m$ equalions

$$
A_{t, k} \mu_{,} \mu_{k}+i_{1} B_{t k} \mu_{k}=0 \quad(i, j, k=1,2, \ldots, m)
$$

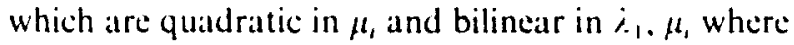

$$
A_{1, k}=\boldsymbol{\Phi}_{c}^{\prime \prime \prime} r_{i} r_{1} l_{k}, \quad B_{t k}=2 \dot{\boldsymbol{d}}_{c}^{\prime \prime} r_{1} r_{k}
$$

By adding to (9) a normalization condition such as, for instance, $\mu_{1} \mu_{1}=1$, a set of $p$ solutions is obtained where, according to the Bezout theorem, $p$ is real at the most equal to $2^{\prime \prime \prime}-1$ and at least equal to one.

By solving eqn (6b), the second order displacements field $u_{z}=l_{p}+\beta_{1} r_{1}$ is obtained, where $r_{p}$, is a particular integral and $\beta_{1}$ arbitrary constants which are determined with $\lambda_{2}$ by imposing the Fredholm condition on eqn $(6 c)$. The following set of lincar equations is obtained:

$$
\left[\begin{array}{c:c}
2 A_{i, k} \mu_{1}+\lambda_{1} B_{i k} & B_{i k} \mu_{i} \\
\hdashline \mu_{i} & 0
\end{array}\right]\left\{\begin{array}{c}
\beta_{i} \\
\hdashline \lambda_{2}
\end{array}\right\}=\left\{\begin{array}{c}
f_{k}\left(\mu_{1}, \lambda_{1}\right) \\
\hdashline g\left(\mu_{i}\right)
\end{array}\right\} .
$$

where $f_{k}$ and $g$ are known functions and the last equation is a normalization condition expressing the orthogonality between $u_{1}$ and $u_{2}$. Equations (11) are solved for each $p$-tuple $\left(\mu_{1}, \lambda_{1}\right)$ and furnish the second order coefficients $\beta_{1}$ and $\lambda_{2}$ for each bifurcated path.

If the system is symmetric, then all coefficients $d_{t k}$ vanish and eqns (9) furnish $\lambda_{1}=0$. 
Coefficients $\mu_{i}$ remain undetermined at this level and are evaluated with $\lambda_{2}$ from the solvability conditions of eqns $(6 c)$ which read

$$
2 A_{i, k l} \mu_{i} \mu_{j} \mu_{i}+\lambda_{2} B_{i k} \mu_{i}=0 \quad(i, j, k, l=1,2, \ldots, m)
$$

to which the condition $\mu_{i} \mu_{i}=1$ has to be added. In eqns (12) it is

$$
A_{i j k l}=\Phi_{c}^{\prime \prime \prime} v_{i} v_{j k} v_{l}+\frac{1}{3} \Phi_{\mathrm{c}}^{\prime \prime \prime \prime} v_{i} v_{j} v_{k} v_{1}
$$

being $u_{2}=\mu_{1} \mu_{k} v_{j k}$ a particular solution of eqn (6b). The coefficients $\beta_{i}$ which are undetermined at this level may be set equal to zero. Equations (12) furnish $1 \leqslant q \leqslant 3^{m}-1$ real solutions. When initial imperfections are present, eqns (9), (11) and (12) are corrected by adding an extra term which accounts for the amplitude imperfections $\xi$ and for the corresponding shape [see Pignataro and Luongo (1988)].

The case which is of interest arises when the system is nearly symmetric. In this case it is $A_{i j k}=O(\chi)$ where $\chi$ is a small parameter and therefore, from eqns (9), it is $\lambda_{1}=O(\chi)$. The matrix of system (11) is thus ill-conditioned and the second order coefficients $\beta_{i}$ and $\lambda_{2}$ tend to infinity, as they are of order $O\left(\chi^{-1}\right)$. Consequently, results furnished by the series expansion (2) are valid in a very small neighborhood of the bifurcation point only; indeed, for $\xi=\mathbf{O}(\chi)$. second order terms are no longer a small correction of the first order terms and the asymptotic series expansion is no more uniformly valid. In addition, for $\chi \rightarrow 0$ the solution of the asymmetric system diverges, thus preventing the recovery of the solution relative to the symmetric case which is governed by the set of cubic eqns (12). This implies the loss of the main feature of the perturbation method in that parametric solutions can no longer be obtained.

It is worthwhile to observe that this drawback arises only in interactive buckling. If there is only one buckling mode, then $\mu_{1}=1$ and eqn (9) furnishes $\lambda_{1}=-A_{111} / B_{11}$. Equations (II) become

$$
\left[\begin{array}{cc}
A_{111} & B_{11} \\
1 & 0
\end{array}\right]\left\{\begin{array}{l}
\beta_{1} \\
\lambda_{2}
\end{array}\right\}=\left\{\begin{array}{l}
f_{1} \\
g
\end{array}\right\},
$$

which are well conditioned even if $A_{111} \rightarrow 0$. Besides, by expliciting $f_{1}$, one can see that the solution approaches that of the symmetric system for $\chi \rightarrow 0$ (Pignataro and Luongo, 1988). The reason for the different behavior of the solutions of the two problems corresponding to $m>1$ and $m=1$ lies in the fact that in the first case, in contrast with single buckling problems, the number and the directions of the bifurcated paths are not known.

\subsection{Modified perturbation method}

From previous discussions on the results of the standard method, it appears desirable to formulate a procedure for nearly symmetric systems which allows one to obtain a solution valid in a wider region around bifurcation, from which the symmetric solution is consistently recovered when $\chi \rightarrow 0$. The following preliminary considerations may guide our reasoning in finding a correct approach for the solution of the problem :

(a) the post-buckling behavior is always governed by third order terms of the energy in a suitably chosen region around bifurcation, the amplitude of which depends on $\chi$;

(b) far from bifurcation fourth order terms become dominant within the domain of interest :

(c) there exists an intermediate region inside which third and fourth order terms of the energy are comparable and therefore both are necessary to describe the mechanical behavior of the system. This implies that in the asymptotic procedure they must appear in the same order perturbation equation. 
Our aim is achieved if we succeed in describing the structure behavior inside the transition region. To this end let us assume that all third order terms are small, of order $\chi$. that is

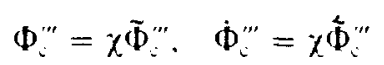

where we define

$$
x=k \zeta
$$

The standard perturbation eqns (6b) are therefore modified by shifting third order terms to the next order equation. By following the same steps as in the previous case, $\lambda_{1}=0$, $u_{z}=0$ are found and the Fredholm conditions are rewritten as

$$
2 \cdot A_{i k k} \mu_{1} \mu_{1} \mu_{1}+i_{2} B_{i k} \mu_{1}+2 \kappa \cdot I_{i, k} \mu_{i} \mu_{1}=0
$$

where $\tilde{A}_{1, k}=\tilde{\Phi}_{c}^{\prime \prime \prime} l_{i} r, l_{k}$. Note that eqns (17) are the same as eqns (12) relative to the symmetric system with an extra term accounting for the weak asymmetry of the structure. In the following we shall refer to eqns (17) as modified perturhation equations. These equations. with the normalization condition $\mu_{1} \mu_{1}=1$, furnish $\mu_{t}$ and $\lambda_{2}$ as a function of the parameter $k$ and therefore of ${ }^{\prime}$. The solution is then written in the form

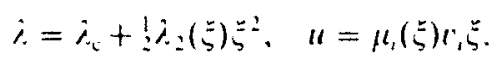

Faced with imperfections it is sulficient to add an extrat term to eyns (17) (1.uongo and Pignatatro, 1988).

Fquations (17) describe the bifurcated paths in a wide domain around bifurcation. They are suited to a numerical solution ats well ats to an asymptotic solution for large values of $k$ (i.c. in alceordance with eqn (16), in the neighborhood of bifurcation) and for small values of $k$ (i.c. far from bifurcation). In this waly the solution can be approximated both by an extrapolation from the origin, which is valid in a small neighborhood of it and by an extrapolation starting from a point far away: the two solutions have then to be matched.

Around bifurcation it is seen that, ats $\mu_{t}=O(1)$, for large values of $k$ we have $\lambda_{2}=O(k)$. The problem is therefore governed in this case by the second and third term in eqns (17) which chatracteriat the asymmetric behavior. The solution can be determined as a perturbation of that corresponding to $h=\infty$ : performing a number of straightforward steps, the standard method results relative to the asymmetric structure are recovered.

Far from bifurcation $k$ assumes small values. In this case the problem is governed by the first two terms of the modified perturbation equation which describe the symmetric behavior of the structure. An asymptotic solution can therefore be obtained as a perturbation of that corresponding to $k=0$. By letting

$$
\lambda_{2}=\lambda_{2}^{0}+h_{1}^{*}+\cdots, \quad \mu_{1}=\mu_{1}^{n}+k \mu_{1}^{*}+\cdots,
$$

the following solution is obtained:

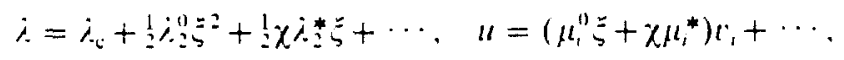

where $\mu_{t}^{0}, \lambda_{2}^{0}$ are solutions of the zeroth order perturbation equations which coincide with eqns (12) and $\mu_{1}^{*}, \lambda_{2}^{*}$ are obtained by solving a lincar problem. Equation (20) furnishes straight lines in the plane of the displacements " which are parallel to those relative to the symmetric system because of the presence of the constant term $\chi \mu_{1}^{*}$. In the limit $\chi \rightarrow 0$ for which the standard procedure fails. the symmetric solution is obtained.

We may conclude that. in the neighborhood of the bifurcation. the structure behavior is described by paths which are near to those of the asymmetric system and successively 
come close to the equilibrium lines of the symmetric structure by remaining far away by a distance of order $\chi$.

It is worth emphasizing that the second order displacements ficld $u_{2}$ is zero as a consequence of the assumption that the third order terms of the energy are small for any. displacements field. Indeed. in the most common cases, the cubic terms of the energy are small only if displacements coincide with the buckling modes. This renders the modified perturbation method applicable only to a restricted class of problems.

\section{ILLUSTRATINE PROBLEM}

The theory has been applied to investigate the post-critical behavior of the two d.o.f. system illustrated in Fig. 3a (Luongo and Pignataro. 1988). Let $\varphi$ and $z$ be the Lagrangian parameters of the system measured as shown in Fig. 3b. The total potential energy can be written as

$$
\Phi=! k_{1} k^{2}+!_{2} k_{2} 3^{2}+l_{2} k_{3} l^{2} \Delta^{2}-N w
$$

where $k_{1}, k_{2}, k_{3}$ are spring constants, $N$ is the vertical load, $w$ the vertical displacement of its point of application and $\Delta$ the stretching of the extensional spring. From kinematics. the following non-linear relations are obtained:

$$
\begin{gathered}
\Delta=\sqrt{(1+\sin p)^{2}+\sin ^{2} p} \\
u=l\left(1-\sqrt{\left.1-\sin ^{2} p-\sin ^{2} p\right) .}\right.
\end{gathered}
$$

If initial imperfections $\vec{p}, \overline{7}$ are present, the energy $(21)$ is modified as

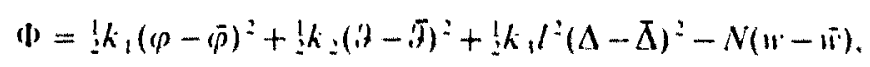

where $X$ and $\bar{n}$ are obtained from (22) and (23) by replacing $\varphi, 2$ with $\bar{p}, \bar{T}$.

By taking the series expansion of eqn (24) up to fourth order terms in $\varphi$ and $t$ and retaining only the bilinear terms $\varphi \bar{p}, 95$ in the initial imperfections we obtain

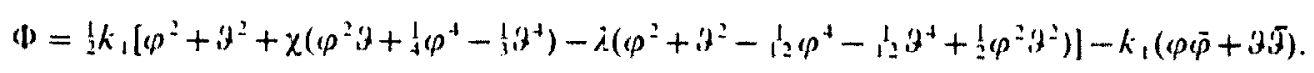
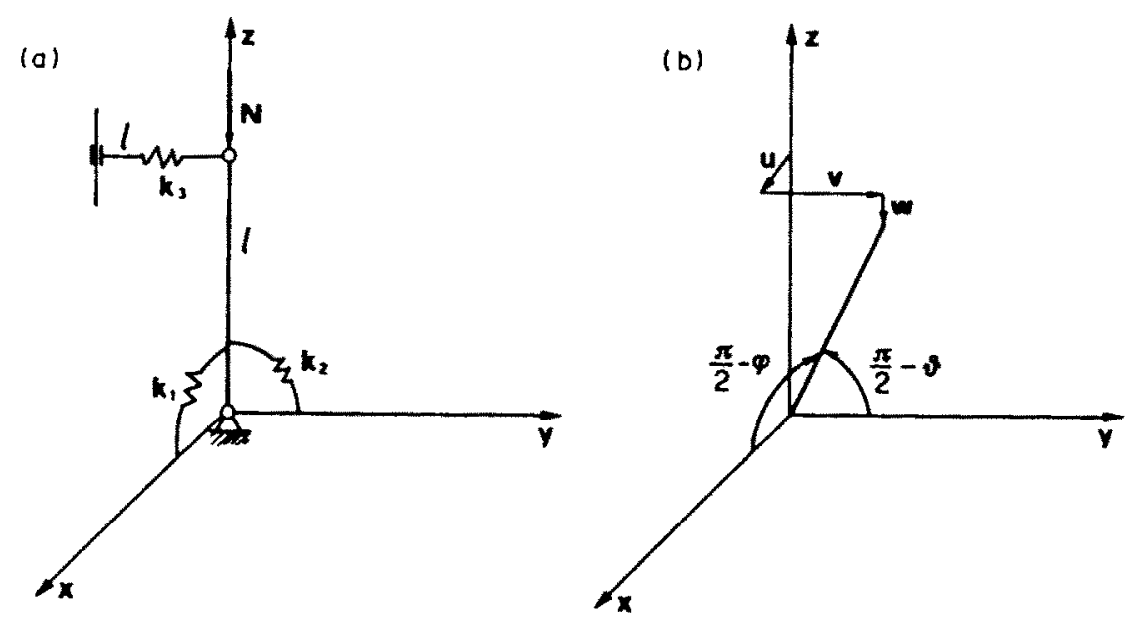

Fig. 3. Two d.o.f. model: (a) reference configuration: (b) varied configuration. 
In eqn (25) $k_{1}=k_{2}+l^{*} k_{3}$ has been taken in order to make the two critical loads to coincide and the following non-dimensional parameters have been introduced:

$$
\chi=l \cdot k_{3} / k_{1}, \quad \lambda=N l k_{1} .
$$

Note that $\Phi[-\varphi, \vartheta]=\Phi[\varphi, \vartheta]$ whereas $\Phi[\varphi,-\vartheta] \neq \Phi[\varphi, \vartheta]$. The asymmetry of the model is due to the extensional spring which is responsible for the cubic terms of the energy proportional to the factor $\chi(0 \leqslant \chi \leqslant 1)$. A family of these models is examined. characterized by different values of $\chi$. by first applying the standard method and then the modified method.

By applying the standard perturbation method (SPM) it is found that the asymmetric perfect system $(\chi \neq 0)$ exhibits $p=3$ post-buckling equilibrium paths depicted in Fig. 4 il in the plane of the Lagrangian parameters:

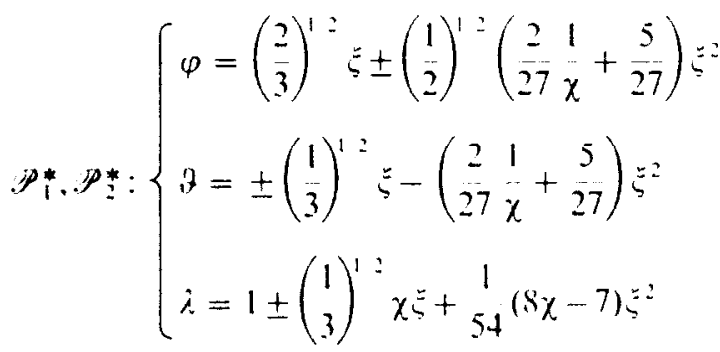

$$
\begin{aligned}
& . P_{3}^{*}: \varphi=0, \quad \lambda=5 . \quad \lambda=1+\frac{1}{1}(1-4 \chi) \xi^{2} .
\end{aligned}
$$

In the same figure curves $\mathscr{P}_{1}, \mathscr{P}_{2}, \mathscr{P}_{1}$ are the linear approximations of curves $\mathscr{P}_{1}^{*}, \mathscr{P}_{2}^{*}, \mathcal{P}^{*}$.

The symmetric system $(\chi=0)$ has instead $q=4$ equilibrium paths represented in Fig. $4 b$

$$
\begin{aligned}
& \mathscr{Y}_{1,2}^{\prime}: \varphi=(1 / 2)^{1: 25}, \quad \lambda= \pm(1 / 2)^{1: 5}, \quad i=1-(1 / 6)^{52} \\
& \mathscr{Y}_{3}: \varphi=\xi, \quad \gamma=0, \quad \lambda=1+(1 / 6) \xi^{\prime} \\
& \mathscr{F}_{4}: \varphi=0, \quad \lambda=\xi, \quad \lambda=1+(1 / 6) \xi^{2} \text {. }
\end{aligned}
$$

Equations (27) and (28) are determined by solving eqns (9), (11) and (12), respectively.

Note that the asymmetric and symmetric systems exhibit a diflerent number of bifurcated paths. In addition the solution (27) is unable to represent the behavior of the synmetric system for $\chi \rightarrow 0$ since second order terms diverge and therefore its validity for small villues of $\chi$ is limited to a small neighborhood of the bifurcation point.

The modified perturbation method (MPM) has heen successively utilized. The relevant modified perturbation equations read
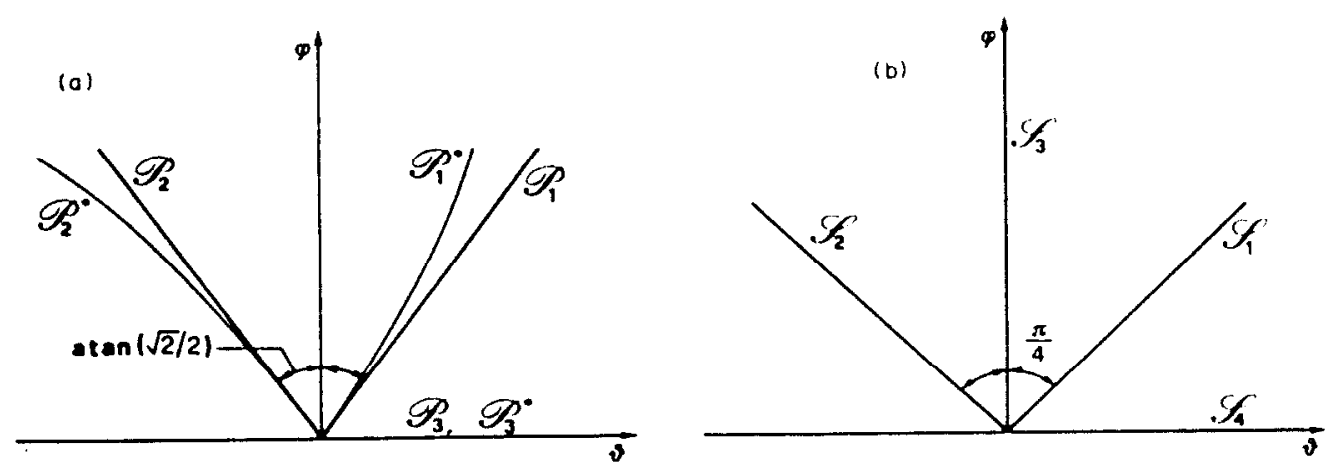

Fig. 4. Bifurcated paths: (a) asymmetric $\operatorname{system}(\chi \neq 0)$; (b) symmetric system $(\chi=0)$. 


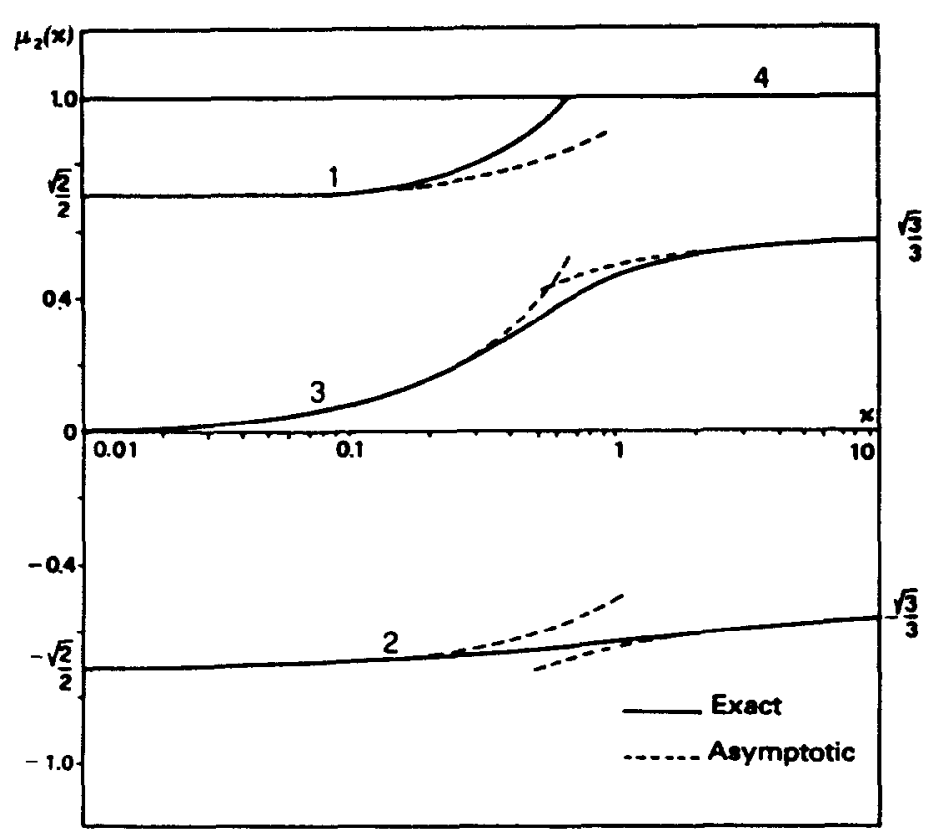

Fig. 5. Solutions $\mu_{2}(x)$ to the moditied perturbation equations.

$$
\begin{aligned}
& i \mu_{1}^{3}-2 \mu_{1} \mu_{2}^{2}-2 \lambda_{2} \mu_{1}+4 \kappa \mu_{1} \mu_{2}=0 \\
& i \mu_{2}^{3}-2 \mu_{1}^{2} \mu_{2}-2 \lambda_{2} \mu_{2}+2 \kappa \mu_{1}^{2}=0 \\
& \mu_{1}^{2}+\mu_{2}^{2}=1 .
\end{aligned}
$$

whose solutions furnish $\mu_{1}(k)$ and $\mu_{2}(k)$. Four solutions are obtained for $\mu_{2}(k)$ and represented in Fig. $5 \mathrm{in} \mathrm{semi-logatrithmic}$ scille. It is seen that for large values of $k$, i.e. according to eqn (16), for small values of $\xi$, three solutions corresponding to those of the standard method for asymmetric systems are obtained; for small values of $\kappa$ (large values of $\xi$ ), the four solutions furnished by the standard method for symmetric systems are recovered. If the non-linear equations (29) are solved asymptotically for large or small values of $\kappa$, the dashed eurves shown in Fig. 5 are obtained. They are a good approximation of the exact solution with the exception of a region around $k=1$, i.e. $\xi=\chi$.

The situation is clearer if the equilibrium paths $\mathscr{R}_{t}(i=1, \ldots, 4)$, corresponding to the solutions $1-4$ in Fig. 5 , are plotted in the $\varphi, 3$ plane and compared with the $\mathscr{P}_{1}$ and $\mathscr{P}_{i}$ curves (Fig. 6). There are three paths $\mathscr{A}$, starting from the bifurcation point whose tangents coincide with curves $\mathscr{P}_{1}$; however they rapidly change direction and become parallel to

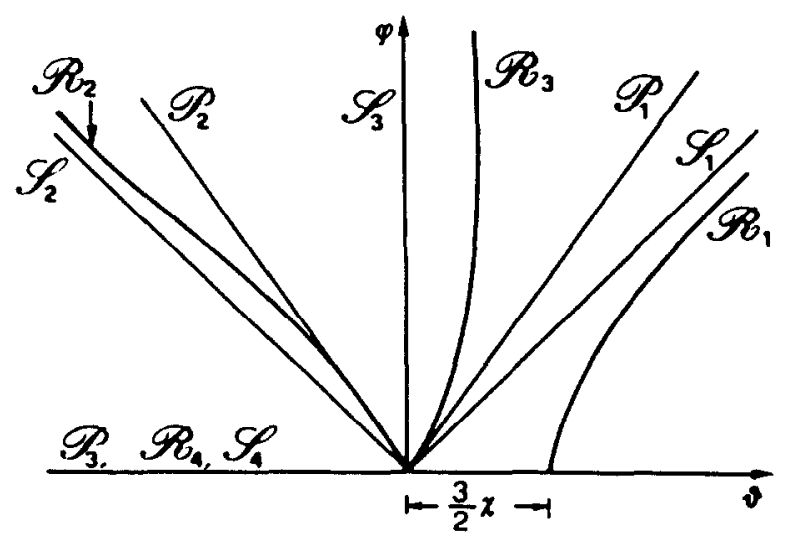

Fig. 6. Bifurcated paths by modified (MPM) and standard (SPM) perturbation methods. 
For this reason, it is more convenient to adopt an alternative approach by following the steps outlined in Byskov and Hutchinson (1979) and Sridharan and Benito (1984). This method. which in many respects is similar to the Koiter original formulation. is more general in that it allows small differences among the buckling loads to be taken into account and, besides, it does not present the drawbacks of the standard method.

The idea of the Galerkin perturbation method (GPM) consists of expressing the displacements field in the form

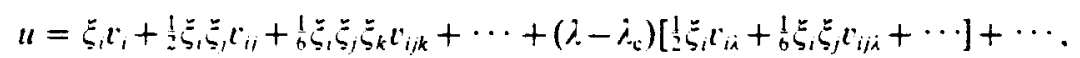

where $\zeta_{i}$ are the amplitudes of the $m$ interacting buckling modes $v_{i}$ and $v_{i j}, v_{i j k}$ are displacements fields which are determined by solving appropriate perturbation equations as specified later. Through a Galerkin approach, the following equilibrium equations are obtained:

$$
\left(1-\frac{\lambda}{\lambda_{\mathrm{ci}}}\right) \xi_{i}+a_{i j k} \bar{\zeta}_{, \xi_{k}}+a_{i, k l} \xi_{,} \xi_{k} \xi_{l}+\cdots=\xi_{i} \quad \text { (no sum with respect to } i \text { ), }
$$

where $i_{s i}(i=1.2, \ldots, m)$ is the critical load associated with the buckling mode $r_{\text {, }}$. Equations (31) can be solved numerically to construct the $\left(\lambda_{.} \xi_{1}: \xi_{1}\right)$ path corresponding to a given initial imperfection $\bar{u}$ assumed as a linear combination $\bar{u}=\sum_{\vec{b}}^{\bar{g}} l_{i}$.

Note that, in contrast with the standard Galerkin method, the displacements field (30) is not expressed as a linear combination of known functions. Indeed, the kinematical description takes into account the evolution of the structure deformation under increasing load in the post-critical range by means of the displacement fiekds $v_{1,}, v_{i, k}, \ldots$ In addition, in the GPM the equilibrium paths are determined by solving the non-linear equations which collect the quadratic and cubic terms all together, thus removing problems pointed out in Section 3 arising in the analysis of nearly-symmetric structures with the SPM.

Dillerences and analogies between the GPM and the SPM become apparent on the basis of the following considerations, relative to the calse $\lambda_{\mathrm{c} i}=\lambda_{\mathrm{v}}(i=1, \ldots m)$. To make our comparative analysis more transparent we start in the two approatches from the same series expansion (2), by temporarily setting aside eyn (30).

In the SPM eqn (5) is sattisfied by forcing each term in $\xi, \xi^{2}, \ldots$ to vanish separately for any kinematically admissible $\delta u$; from this perturbation equations (6) are obtained. By applying the Fredholm condition to each perturbation equation, a relation is established between coeflicients of the same order of the load and displacement series expansion; for instance eqn (9) forges a relationship between $\lambda_{1}$ and $u_{1}$, eqn (11) between $\lambda_{2}$ and $u_{2}$, through the arbitrary constants $\mu_{\text {, and }} \beta_{1}$, respectively.

In the GPM no such relations are established and eqns (6) are solved for arbitrary. ralues of the leat. Due to the singularity of the operator $\Phi^{\prime \prime}$, the solvability of the equations is ensured by introducing a constraint on the displacements field $\delta u$, that is by solving eqns (6) in a subspace of the kinematically admissible functions. By insisting, for instance, that $u_{2}, u_{3}, \ldots$ be orthogonal to each buckling mode $v_{k}$ through a positive definite bilincar operator $T, T i_{k} u_{2}=0, \ldots$, it must be in eqns $(6) T v_{k} \delta u=0$. We now observe that, in virtue of eyn (8). ecyn (6b) admits the solution

$$
u_{2}=\mu_{1} \mu_{1} v_{11}+i_{1} \mu_{1} v_{i \lambda}
$$

where $t_{t}$ and $t_{t a}$ are solutions of the variational problems

$$
\begin{aligned}
& \Phi_{c}^{\prime \prime} v_{i j} \delta u=-\Phi_{i}^{\prime \prime \prime} v_{i} v_{j} \delta u \\
& \Phi_{c}^{\prime \prime} v_{i j} \delta u=-2 \Phi_{c}^{\prime \prime} v_{i} \delta u,
\end{aligned}
$$

under the conditions 
Equations (33) and (34) can be solved through a Lagrange multiplier technique. In contrast with the SPM. the coefficient $\lambda_{1}$ in eqn (32) is undetermined.

It is worth noticing that in this approach the orthogonality condition plays a fundamental role in that it ensures the solvability of the problem. On the contrary, in the Budiansky procedure, it serves as a normalization condition only.

At this stage of the procedure, if one substitutes eqn (8) for $u_{1}$, eqn (32) for $u_{2}$ etc. into (2a) the series expansion (30) is obtained, if one poses $\xi \mu_{t}=\xi_{i}$ and takes from eqn (2b) $i_{1}=i_{i}=i_{\text {. }}$.

By introducing $u_{1}, u_{z}$ etc. into eqn (5), the equilibrium equation is satisfied for any $\dot{\delta}_{u}$ orthogonal to $v_{k}(i=1, \ldots m)$. By requesting the equation to be satisfied for $\delta u=v_{k}$ also. the equilibrium equations (31) are finally obtained in the modes with amplitudes $\xi_{k}=\xi \mu_{k}$ and in the load parameter $i_{-}-\lambda_{x}=\lambda_{1} \xi+\cdots$. The last step of the method formally coincides with a procedure of the Galerkin type where the function $u$ is expressed through eqn (30) and the test function $\delta u$ as a variation of the terms linear in $\xi$ onl $l_{j}, \delta u=v_{k} \delta \xi_{k}$. Indeed, higher order terms in 5 appearing in $\delta u$ do not play any role in eqn (5), due to the particular choice of $u_{2}, u_{1}$.

Finally, we want to show how the GPM can advantageously be utilized in the analysis of nearly symmetric systems. The basic idea consists of expressing all quantities as a perturbation of those of an arbitrarily chosen symmetric system through a series expansion in terms of the asymmetry parameter $\chi$. It is obviously convenient to choose the symmetric system in such a way ats to make the solution of the problem as simple as possible. By proceding in this way the total potential energy and the displacements field are written as

$$
\begin{aligned}
D[u ; \lambda ; \chi] & =\mathbb{w}_{0}[u ; \lambda]+\chi \tilde{w}[u ; \lambda]+\mathrm{O}\left(\chi^{2}\right) \\
u(\chi) & =u^{0}+\chi \tilde{u}+\mathrm{O}\left(\chi^{2}\right),
\end{aligned}
$$

where $D_{0}=(1)(u, \lambda ; 0), u^{\prime \prime}=u(0), \tilde{\Phi}=\left(\partial(D / \partial \chi)_{x}-0, \tilde{u}=(\partial u / \partial \chi)_{x}-0\right.$.

The eigenvalue problem (6a) now furnishes the solution

$$
u_{1}=\mu_{i}\left(v_{i}^{\prime \prime}+\chi \tilde{i}_{1}\right)
$$

where $v_{i}^{i \prime}$ are $m$ simultaneous buckling modes of the symmetric system $\omega_{i k}^{*} c_{1}^{0 \prime} \delta u=0$ and the corrections $\tilde{i}_{i}$ are solutions of the problem

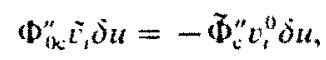

under the auxiliary conditions

$$
T v_{k}^{\prime \prime} \dot{j} u=0 \quad(k=1,2, \ldots, m) .
$$

The second order displacements field is now

$$
u_{2}=\mu_{i} \mu_{i}\left(v_{i j}^{0}+\chi \bar{c}_{i j}\right)+\hat{\lambda}_{1} \mu_{i}\left(v_{i \alpha}^{0}+\chi \bar{v}_{i \alpha}\right)
$$

where $v_{i j}^{\prime \prime}$ and $v_{i k}^{13}$ are the secondary modes of the symmetric system which satisfy eqns (33), (34) whereas the corrections $i_{i j}$ and $i_{i x}$ are determined by solving the variational problems

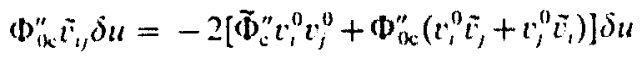

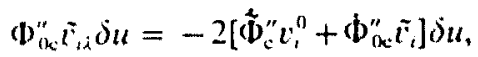

under the constraints (38).

In conclusion. once the displacements of the symmetric system have been determined. the corresponding corrections which characterize the nearly symmetric system are evaluated 
by solving a sequence of linear problems where the stiffness operator is the simpler one of the symmetric structure. Then the non-linear equilibrium equations as previously illustrated are derived.

\section{CONCLUSIONS}

The non-linear interaction between several buckling modes in nearly symmetric structures has been investigated. The interest has been focused on a perturbation method capable of correctly describing the non-linear equilibrium paths of the perfect and imperfect structures. It has been shown that the standard perturbation method formulated by Budiansky fails whenever a small parameter $\chi$ which describes the asymmetry of the system approaches zero, thus preventing the recovery of the solution of the symmetric system. This drawback can be overcome for a restricted class of structures by shifting cubic terms which are responsible for the asymmetry behavior from second order to third order perturbation equations.

A system with two degrees of freedom has served to explain the problem and to show the role played by post-bifurcated paths in the description of the mechanical behavior of the structure.

An alternative method which follows the Galerkin approach has then been discussed by showing differences and analogies with the standard method. It is seen that the Galerkin method is more general and does not exhibit the drawbacks of the standard method in that the load displacements law is determined by solving equations where all non-linearities of the problem appear at the same level. The method can then be specialized to analyze nearly symmetric structures by considering the actual system as a perturbation of a properly chosen symmetric system. In this way, the displacement fields are obtained by solving linear equations where the stiffness operator is the simpler one of the symmetric system.

\section{REFFERENCES}

Bentito, R. and Sridharan, S. (1985). Mede interaction in thin-walled structural members. J. Struct. Mech. 12(4), $5175+2$.

Bradford. M. A. and tlanecck, G. J. (1984). Flastic interaction of local and lateral buckling in beams. ThinHalled Siructures 2(1), 125

Hudiansky. B. (1974). Theory of buckling and post-buckling behaviour of elastic structures. In Advances in Applied Mochenics (Edited by Chiat-Shun Yih), Vol. 14, pp. I-65. Academic Press, New York.

Byskov. E., Ditmkilde, L. and Jonsen, K. J. (1989). Multimode interaction in axially stiffened cylindrical shells. Mech. Struct. Mach. 16(3), 387.405.

Hyskuv, R. and Hutchinson, J. W. (1979). Mode interaction in axially stiffened cylindrical shells. $A / A A J / 17(6)$, 6306.33

Elisliakotr, I. (1980). Remarks on the static and dynamic imperfection-sensitivity of nonsymmetric structures. J. Appl. Hech. 47.111 115.

Koviter, W. T. (19+5). Over de stabiliteit van het elaststisch evenwicht (in Dutch), PhD Thesis, H. J. Paris, Amsterdam : English translation as NASA TT F-10, 833, 1957 and AFFDL Report TR 70-25, 1970.

Luongo, A. and Pignitaro. M. (1988). A nonstandard perturbation analysis of the interactive buckling in nearly symmetric systems. Dipartimento di Ingegneria Strutturale e Geotecnica, University of Rome "La Sapienza", R.port No. 7

Luongo, A. and Pignataro. M. (1989). Multiple interaction and localization phenomena in postbuckling of compressed thin-walled members. AlAA Jl 26(II), 1395-1402.

Nayfith. A. (1973). Perturhation Methods. Wiley, New York.

Pignataro, M. and Luongo, A. (1987). Asymmetric interactive buckling of thin-walled columns with initial imperfections. Thin-Wulled Structurex 5, 365-386.

Pignatiro, M. and Luongo, A. (1988). General treatment of interactive buckling in imperfect structures. Dipartimento di Ingegneria delle Strutture, University of L'Aquila, Report No. 6.

Pignataro, M., Luongo, $A$. and Rizzi. N. (1985). On the effect of the local-overall interaction on the postbuckling of uniformly compressed channels. Thin-Walled Structures 3, 293-321.

Sridharan, S. and Ali. M. A. (1985). Interactive buckling in thin-walled beam columns. ASCE 111, EM12, 14701486

Sridharan, S. and Benito, R. (1984). Columns: static and dynamic interactive buckling. ASCE 110, EM1, 49-65. 